\title{
Implementing Data - The Future of Alternative Methods - VZET Symposium 2019
}

doi:10.14573/altex.1906211

The third Symposium of the Virtual Center for Replacement/ Complementary Methods to Animal Testing (VZET) was held at the University of Veterinary Medicine Hannover, Foundation (TiHo), in Hannover, Germany on March 27-28, 2019 to celebrate the tenth anniversary of the VZET. The Center was established to optimize method development and teaching in the field of veterinary medicine based on the 3Rs. Members of the TiHo-based VZET are involved in the R2N consortium (Replace and Reduce in Lower Saxony), which aims to implement alternative methods in biomedical research. Bringing together scientists from different backgrounds, the VZET Symposium promoted a broad scientific exchange on the 3 Rs field.

Dr Katherina Sewald (Department of Preclinical Pharmacology and In-Vitro Toxicology, Fraunhofer ITEM, Hannover, Germany) emphasized the advantages of using human lung tissue in early drug development. She gave an overview of how the use of human tissue is being implemented in research areas like asthma, allergy, COPD, respiratory function and pulmonary fibrosis and referred to the importance of validation and quality assessment of the tissues. She also addressed the difficulties associated with the acquisition of human probes. The fact that there are differences between species requires a change to the dogma that animal experimentation is the best reference point for biomedical research. The predictive validity of models for humans can be improved by basing them on human tissue.

Dr Helena Kandarova (Slovak Toxicology Society SETOX, Bratislava, Slovak Republic) elaborated on three-dimensional reconstructed human skin models and their possible applications in regulatory use and biomedical research. She highlighted the similarities to human skin but also pointed out differences (e.g., the absence of certain lipid classes in the stratum corneum). Reconstructed human skin equivalents are well accepted in the field of toxicological skin corrosion and skin irritation testing under the REACH (Registration, Evaluation, Authorization of Chemicals) legislation and for preclinical testing of drug phototoxicity (ICH S10). These skin models are now also being considered for implementation in the ISO guidelines for the testing of medical devices. Dr Kandarova demonstrated the benefits of reconstructed human skin models for endpoints such as skin irritation, wound healing studies and therapies.

Prof. Ellen Fritsche (IUF - Leibniz Research Institute for Environmental Medicine, Düsseldorf, Germany) gave insights into adverse outcome-based developmental neurotoxicity testing (DNT) and its implementation into the OECD framework for regulatory applications. Prof. Fritsche stressed that the transfer from preclinical studies with rodents to clinical trials in humans often fails due to differences between species. This learning has further led to a paradigm shift in toxicity testing, the use of human cells, and mode of action-based approaches. Due to uncertainties in methodology, evaluation and regulation of in vivo DNT testing strategies, researchers are developing new in vitro tools. The software-supported analysis of the migration and differentiation to neurons and glia cells of human induced pluripotent stem cell-derived neural progenitor cells, the so-called "Neurosphere Assay", is an important element of the DNT testing battery. However, adverse outcome pathways in the field of DNT are still lacking and will have to be expanded in the future.

Dr Winfried Neuhaus (Austrian Institute of Technology, Department Health and Environment, Molecular Diagnostics, Vienna, Austria) discussed in vitro blood-brain-barrier (BBB) models, the cell types used in the models, and the importance of physiological paracellular tightness. He stated that there is no "perfect" BBB model and that the most suitable model is "as easy as possible and as complex as necessary". The methods need to be validated for each individual application.

Prof. Jan G. Hengstler (IfADo - Leibniz Research Centre for Working Environment and Human Factors, TU Dortmund University, Dortmund, Germany) presented a new method to predict drug-induced liver injury (DILI) in humans. He pointed out the shortcomings of extrapolation from animal experiments to human patients with regard to DILI. In order to overcome these disadvantages in the identification of substances inducing DILI, an in vitro/in silico method was developed, which models data based on the maximal blood concentration of the substances. These new prediction methods could improve DILI prediction in vivo.

In addition to the key note lectures, doctoral students, postdoctoral fellows, and principal investigators presented data from their current projects in short talks. The focus was on alternative methods in the field of veterinary relevant infections, neurodevelopmental and neurotoxicity testing, as well as botulinum neurotoxin potency testing, BBB models, and use of advanced extracellular matrices in cell culture.

In the plenary discussion, moderated by Prof. Gerhard Breves, chairman of the VZET, important key questions around alternatives to animal testing were discussed. The keynote speakers discussed that in vitro methods cannot be expected to replace animal experiments on a 1:1 basis in most cases. Therefore, work to reduce the number of animals and to gain mechanistic insights is of great value where there are no approaches yet to completely replace animal testing. The choice of model should take the target 
species into consideration. Data integration and implementation of human tissues and cells in cell culture has great importance in current scientific work and increases predictive power of experiments analogous to animal experiments. In vitro methods often have the disadvantage that they need to be validated against animal experiments, the so-called "gold standard", which may not be the best choice for an extrapolation to human data. In vitro models have their strengths in elucidating molecular mechanisms and can offer added value for defined questions.

\section{Bettina Seeger}

Institute for Food Toxicology and Virtual Center for Replacement/

Complementary Methods to Animal Testing

University of Veterinary Medicine, Hannover, Germany

\section{Meeting Report The Biological Evaluation of Medical Devices:
Transition to 2017/745 MDR in Progress}

doi:10.14573/altex.1907011

On May 23, 2019 VitroScreen organized a seminar on the biological evaluation of medical devices (MD) focusing on biocompatibility aspects (cytotoxicity, irritation, and sensitization potential). MDs are an heterogenous and complex class of products whose legislative framework has recently been revised with the introduction of EU Regulation 2017/745 (MDR). Being a key step within the assessment and risk management process, the biological evaluation has to be properly addressed and documented in the dossier of each device entering the market. Currently, there are no harmonized standards and common specifications available to drive the transition from the old MD Directive 93/42/CEE to the new MDR and a large number of questions have arisen from industry as well as from the Notified Bodies involved in the CE marking process.

Dr Marisa Meloni, CEO of VitroScreen, welcomed the participants and highlighted the importance of gathering together representatives of Notified Bodies (ISS, ITALCERT, Kiwa Cermet, and others), as well as toxicologists and regulatory toxicology experts, members of the ISO/TC 194 Working Group, and regulatory affairs specialists operating in the industry and academia in a think tank to address hazard and risk assessment issues in the medical device sector. This allowed to discuss the difficulties being encountered, to share experiences and expertise, and to formulate a vision for the future of medical device evaluation in order to move forward towards a robust toxicological approach by overcoming the difficulties and uncertainties in the transition period. The seminar offered a unique opportunity to achieve a better understanding of the evolution of the ISO 10993 standard and the applicability of OECD validated in vitro test methods and non-animal testing approaches to the assessment of the skin irritation and skin sensitization potential of medical device extracts and substance-based devices. It was underlined that the ISO standard is evolving not only to respond to regulatory requirements but also to the ethical and scientific concerns which demand the replacement of animal testing and the adoption of more reliable and predictive testing strategies according to Directive 2010/63/ EU.

Dr Christian Pellevoisin (EpiSkin, Convenor of ISO Group 8, Member of Technical Committee 194 (ISO/TC 194/WG8)) delivered a lecture entitled "Updates to future ISO 10993-23 standard: in vitro assessments to support biocompatibility and perspectives for sensitizing potential" underlining the significant progress achieved in the evaluation of skin irritation and skin sensitization potential in the medical device sector at ISO level. Skin irritation testing is one of three biocompatibility tests recommended for all medical devices (ISO 10993-1), the others being skin sensitization and cytotoxicity. According to ISO 10993-10 "Biological evaluation of medical devices - Part 10: Tests for irritation and skin sensitization", some in vitro approaches do exist for this endpoint but they are only validated for neat chemicals and not for medical device extracts. Hence, the Draize test performed in vivo on rabbits is still requested. Knowing both the ethical and scientific limits of animal approaches, the ISO TC194/WG8 initiated a project to adapt the existing in vitro OECD protocol for chemicals (TG 439) to medical device (MD) products in 2012. The OECD TG 439 for skin irritation of chemicals is based on reconstructed human epidermis models $(\mathrm{RhE})$. Validated $\mathrm{RhE}$ models have undergone long and strict evaluation processes by centers of validation of alternative methods (ECVAM, JaCVAM) before their acceptance. The robustness, quality, and stability of the RhE models as well as the transferability and predictability of the methods have been challenged with large sets of chemicals in at least 3 different laboratories. Then, the results were peer-reviewed by an independent scientific committee before being considered for inclusion in TG 439. Considering this and the recent guideline for good in vitro methods from OECD (GIVIMP), it has been decided to rely only on RhEs previously validated in an OECD TG to develop a protocol adapted to medical devices. Two models, SkinEthic RHE from EPISKIN (FR) and Epiderm from MatTek (US) have been used to develop a method to assess 